\title{
Analysis of Channel Model for GEO Satellite Mobile Communication System
}

\author{
$\mathrm{Lu} \mathrm{Lu}$ \\ Institute of Communication Engineering, PLA University \\ of Science and Technology \\ Nanjing, 210007, China \\ katherine10@163.com
}

Aijun Liu

Institute of Communication Engineering, PLA University

of Science and Technology

Nanjing, 210007, China

\begin{abstract}
In GEO satellite mobile communication system, a line-of-sight (LOS) component exists as well as multipath component, which are influenced by buildings or foliage that causing shadowing effect. Satellite mobile channel is described as fading model based on Markov process for channel properties in large circumstance. In this paper, a fading channel model based on three-state semi-Markov process is analyzed which several common models are taken into consideration. For received signals, multipath fading, shadowing effect and Doppler shift need to be discussed. This model is built on Rician model, Rayleigh model and C.Loo model, the probability density function (PDF) statistical properties are given, the functions of CCDF, LCR and ADF are derived. Simulation results suggest that this model switches among the three states obeying semi-Markov process, it sufficiently reflexes the merits of mixed channel model, provides an important meaning for channel actual tests.
\end{abstract}

Keywords-Satellite mobile communication system; fading channel; semi-Markov process; three-state switch

\section{INTRODUCTION}

Satellite mobile communication is widely used by subscribers for its offering communication services using satellite as relay. It is becoming a hot point in communication field, and the research of channel transmission properties and channel model are the significant aspects for engineering exploitation and design of satellite mobile communication system. Satellite mobile communication channel has the properties both in satellite and mobile channels, such as fading, multipath effect, Doppler shift, etc[1]. For the past many years of measure, lots of satellite mobile channel models had been put forward, C.Loo model[2], Lutz model[3], Corazza model[4] are the common channel model for transmission statistical probability. Generally speaking, channel statistical models divide into single model and mixed model, single for simplify procedure and improve efficiency, mixed uses weighting prediction method to describe transmission channel.

In recent years, many scholars have suggested new satellite

\author{
Daoxing Guo \\ Institute of Communication Engineering, PLA University \\ of Science and Technology \\ Nanjing, 210007, China \\ Maoqiang Yang \\ Institute of Communication Engineering, PLA University \\ of Science and Technology \\ Nanjing, 210007, China
}

mobile channel model for accurately channel property. Fernando and his partners put forward a channel model based on Markov process[5], giving the reality measure for the model statistical parameters. Jose Luis[6] and his partners presented a model with states switching based on Markov process, overcoming the drawbacks under traditional single state for describing large circumstance surroundings.

In this paper, a three-state channel model based on semi-Markov process is described which is presented based on mixed channel model. The structure of the paper is organized as follows: Section II describes the channel properties causing signal fading. In Section III, we present the definition of three-state semi-Markov process, discussing the switch among the three different states. Simulation results are displayed in Section IV, verifying the effectiveness of the proposed model. Finally in Section V, some conclusions are drawn in this paper.

\section{CHANNEL PROPERTIES}

In satellite communication system, received signal is given as

$$
\begin{aligned}
c(t) & =s_{1}+s_{2}+s(t) \\
& =A_{0} e^{j\left(2 \pi f_{0} t+\varphi\right)}+\sum_{m=1}^{M} A_{m} e^{j\left(2 \pi f_{m} t+\varphi_{m}\right)}+s(t)
\end{aligned}
$$

Where $s_{1}$ is the line-of-sight (LOS) component, going through slow fading; $A_{0}, f_{0}$ and $\varphi$ are amplitude, Doppler shift and phase; $s_{2}$ is multipath component causing fast fading; $s(t)$ is Gauss white noise.

\section{A. Multipath fading}

Transmitting signals may be obstructed by foliage or construction, resulting in diffraction, reflection and dispersion. Received signals are the mixture from multipath, which is called multipath fading. Multipath fading usually described by 
Rayleigh distribution, the probability density function (PDF) of signal envelope is

$$
f_{r}(r)=\frac{r}{\sigma^{2}} \exp \left(-\frac{r^{2}}{2 \sigma^{2}}\right)
$$

Where $\sigma$ is average dispersion power caused by multipath fading.

\section{B. Shadowing effect}

Obstructions on signal propagation path result in signal level dropping, when LOS absorbed or dispersed by obstructions, shadowing effect occurred. Shadowing effect usually described by lognormal distribution, the PDF of signal envelope is

$$
f_{r}(r)=\frac{1}{r \sigma \sqrt{2 \pi}} \exp \left[-\frac{(\ln r-\mu)^{2}}{2 \sigma^{2}}\right]
$$

Where $\mu$ and $\sigma$ are logarithmic mean and variance of LOS amplitude.

\section{Doppler shift}

The change of location causes Doppler shift and time selective fading. For GEO satellite mobile communication system, satellite remains relatively static, the Doppler shift is indicated by

$$
f_{d}=f \frac{v}{c} \cos \theta_{0} \cos \theta_{e}
$$

Where $\theta_{0}$ denotes the intersection angle between the drop shadow of satellite-user link and the moving direction of user, $\theta_{e}$ denotes the elevation of the subscriber location, $v$ denotes the moving speed of user. The relationship is shown in Fig.1.

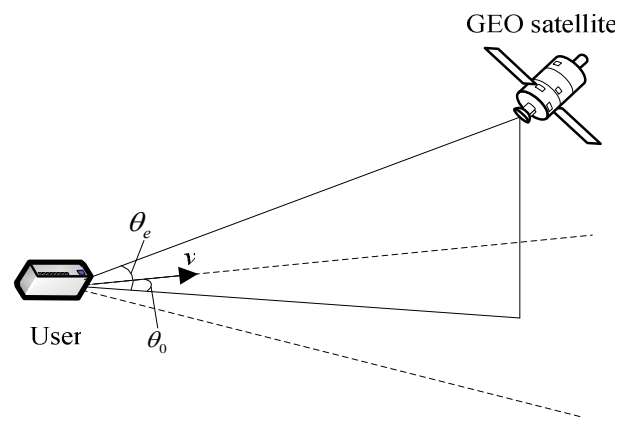

Fig.1. Doppler shift

\section{CHANNEL MODEL BASED ON SEMI-MARKOV PROCESS}

Receiving signals are the addition of LOS component and multipath component, the envelope of receiving signals yield by whether signals are fading or not. Based on the fade duration in ITU-R Recommendation, three types of fading channels are defined, A state: LOS condition; B state: moderate shadowing condition; C state: deep shadowing condition.

\section{A. Three-state semi-Markov process}

The change among channel states is a relatively slow process, it is decided by the distance of user moving process and elevation change, each state keeps a certain distance. It is observed in [5] that this distance is about $5 \mathrm{~m}$, the change of states is described by Markov process. When mobile terminal moves to the certain distance, it switches to a new state at random.

As for three-Markov process, the modeling is indicated in Fig.2.

Where $S_{1}, S_{2}, S_{3}$ denotes respective channel state, $P_{i j}$ is the probability the Markov process goes from state $i$ to state $j$. The

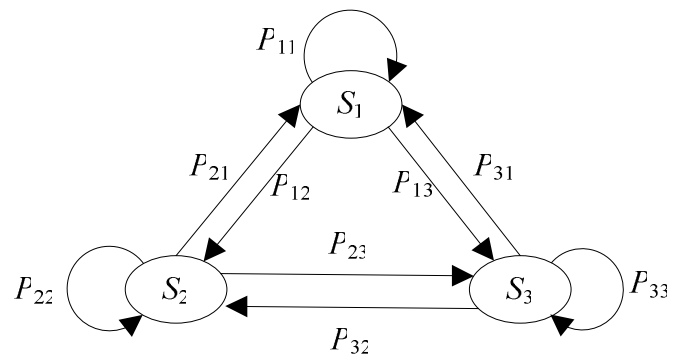

Fig.2. Switching of three-state Markov process

switching among each state is described by a transition matrix $\mathbf{P}$, which is

$$
P=\left(\begin{array}{lll}
p_{11} & p_{12} & p_{13} \\
p_{21} & p_{22} & p_{23} \\
p_{31} & p_{32} & p_{33}
\end{array}\right)
$$

Based on the properties of Markov process, a stationary state vector $\pi$ is calculated as defined, $\vec{\pi} P=P$, then

$$
\pi=\left(\begin{array}{lll}
\pi_{1} & \pi_{2} & \pi_{3}
\end{array}\right)
$$

Semi-Markov process is a Markov chain which the time among switch of each state is random, and states are defined by kinds of distribution [5][7]. The switching process is denoted by a matrix $\mathbf{r}$, where

$$
r_{i j}=\frac{P_{i j}}{1-P_{i i}} \quad \text { for } \quad r \neq j \quad \text { and } \quad r_{i i}=0
$$

The semi-Markov process transition of three states is assumed in Fig.3.

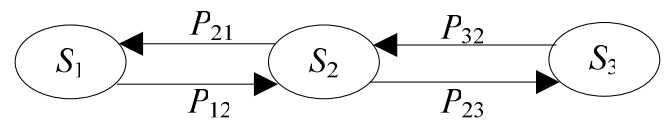

Fig.3. Switching of three-state semi-Markov process 


\section{B. Channel model analysis}

Mixed channel model describes the statistically properties in several different statistical distribution, the merits are sufficiently reflected in non-stationary channel [8]. In this paper, mixed channel is assumed which is described by three irrelevant states switched by semi-Markov process and each is introduced below.

\section{1) Rician model}

When the transmission of LOS component is not blocked by obstructions, it is referred as ideal state. LOS component is not influenced by shadowing effect, the whole receiving signals are the addition of LOS component and multipath component obeyed by Rayleigh distribution and the envelope is obeyed by Rician distribution where Rician factor is $K$. Channel multi-fading coefficient $\alpha \sim C N\left(\mu_{\alpha}, \sigma_{\alpha}{ }^{2}\right)$, the probability density function(PDF) of $\alpha$ is

$$
f(\alpha)=\frac{1}{\pi \sigma_{\alpha}^{2}} \exp \left\{-\frac{\left|\alpha-\mu_{\alpha}\right|^{2}}{\sigma_{\alpha}^{2}}\right\}
$$

The PDF of envelope $\boldsymbol{r}$ is

$$
f(r)=\frac{2 r}{\sigma_{\alpha}^{2}} \exp \left[-\frac{r^{2}}{\sigma_{\alpha}^{2}}-K\right] I_{0}\left(2 r \sqrt{\frac{K}{\sigma_{\alpha}^{2}}}\right) \quad r \geq 0
$$

Where

$$
K=\left|\mu_{\alpha}\right|^{2} / \sigma_{\alpha}^{2}
$$

It is assumed by these expressions that the smaller of $K$, the larger of LOS component average power relative to multipath component, the severer of channel fading. Experiments testing proved in the open air of approximately ideal state, the Rician factor $K$ of satellite mobile channel is between $10 \sim 20 \mathrm{~dB}$.

\section{2) Rayleigh distribution}

When signals are influenced by buildings or foliage, the path between subscriber and satellite is entirely blocked. This condition is referred as deep shadowing state, the propagation of signals are based on stray multipath component. Users receive signals from different directions with random amplitude and phase, which obey Rayleigh distribution, Channel multi-fading coefficient $\alpha \sim C N\left(0, \sigma_{\alpha}^{2}\right)$, the probability density function(PDF) of $\alpha$ is

$$
f(\alpha)=\frac{1}{\pi \sigma_{\alpha}^{2}} \exp \left\{-\frac{|\alpha|^{2}}{\sigma_{\alpha}^{2}}\right\}
$$

The PDF of envelope $\boldsymbol{r}$ is

$$
f(r)=\frac{2 r}{\sigma_{\alpha}^{2}} \exp \left[-\frac{r^{2}}{\sigma_{\alpha}^{2}}\right] \quad r \geq 0
$$

\section{3) Loo model}

In rural or suburb circumstance, as for the existing of trees and small buildings, received LOS component is influenced but not entirely blocked. The receive signals are conducted of LOS condition influenced by shadowing effect and multipath component which is described by Loo model.

Loo model is first put forward by C.Loo in 1985, it mainly aimed at rural circumstance, the receive signals are

$$
r(t)=s(t) z(t)+d(t)
$$

Where $s(t)$ denotes LOS component, $z(t)$ denotes fading by shadow, $d(t)$ denote multipath component produced by dispersion diffraction or reflection.

When the $s(t)$ of LOS component remain fixedness, receiving signals envelope $\boldsymbol{r}$ obey Rician distribution,

$$
f(r \mid s)=\frac{r}{\sigma_{d}^{2}} e^{\frac{\left(r^{2}+s^{2}\right)}{-2 \sigma_{d}^{2}}} I_{0}\left(\frac{r s}{\sigma_{d}^{2}}\right)
$$

Where $I_{0}\left(\mathrm{)}\right.$ is zero-order Bessel function, $\sigma_{d}^{2}$ is average power of multipath component.

As is influenced by shadowing effect, LOS component $s(t)$ obeyed by lognormal distribution

$$
f(s)=\frac{1}{\sqrt{2 \pi} \sigma s} \exp \left[-\frac{\left(\ln s-\mu^{2}\right)}{2 \sigma^{2}}\right], \quad s>0
$$

The PDF of receiving signals envelope $\boldsymbol{r}$ is

$$
\begin{aligned}
f(r) & =\int_{0}^{\infty} p(r \mid s) p_{S}(s) d s \\
& =\frac{r}{b_{0} \sqrt{2 \pi d_{0}}} \int_{0}^{\infty} \frac{1}{s} \exp \left[-\frac{(\ln s-\mu)^{2}}{2 d_{0}}-\frac{r^{2}+s^{2}}{2 b_{0}}\right] I_{0}\left(\frac{r s}{b_{0}}\right) d s
\end{aligned}
$$

The complementary cumulative distribution function (CCDF) of received signal is

$$
F(r)=P(r \leq R)=\int_{0}^{R} f(r) d r
$$

Where $R$ is a certain level value.

Take Doppler shift as consideration, Loo simulation model is indicated in Fig.4. 


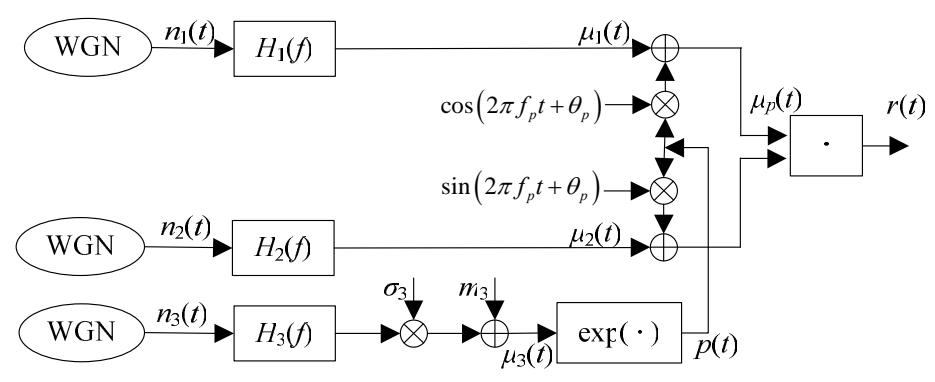

Fig.4. Loo simulation model

Level crossing rate(LCR) and average fade duration(AFD) are two significant high-level statistic, LCR is the expectation which the signal envelope $r$ get through the appointed level with positive slope.

$$
\begin{aligned}
N(r)= & \int_{0}^{\infty} \dot{s} p(r, \dot{s}) d \dot{s} \\
= & \frac{r \sqrt{2 \beta}}{\pi^{3 / 2} \varphi_{0}} e^{-\frac{r^{2}+y^{2}}{2 \varphi_{0}}} \cdot \int_{0}^{\pi / 2} \cosh \left(\frac{r y}{\varphi_{0}} \cos \theta\right) \\
& \cdot\left\{e^{-(\alpha y \sin \theta)^{2}}+\sqrt{\pi} \alpha y \sin (\theta) \operatorname{erf}(\alpha y \sin \theta)\right\} d \theta
\end{aligned}
$$

Where $p(r, \dot{s})$ denotes the joint probability density of $\dot{S}$ and $s$ on the point $s=r, \alpha$ and $\beta$ are defined by Doppler power spectrum density and Doppler frequency.

AFD is the statistic mean value of time interval where signal envelope is under the given level $r$.

$$
T(r)=\frac{p(r)}{N(r)}
$$

Where

$$
p(r)=p\{\eta(t) \leq r\}=\int_{0}^{r} p(s) d s
$$

\section{SIMULATION RESULTS AND DISCUSSIONS}

LOS signal fading is taken into account when we simulate rural or suburb circumstance adopted Loo model, C.Loo and his partners gave model parameters based on Olympus star. As for the analysis of C.Loo model simulation, parameters are given in [9] which is showed in Tab.1.

Tab.1. Loo simulation parameters

\begin{tabular}{|c|c|c|c|c|c|}
\hline $\begin{array}{c}\text { Fading } \\
\text { area }\end{array}$ & $\sigma_{1}$ & $\sigma_{2}$ & $\sigma_{3}$ & $m_{3}$ & $f_{p} / f_{\max }$ \\
\hline heavy & 0 & 0.3856 & 0.5349 & -1.593 & 0.1857 \\
\hline light & 0.404 & 0.4785 & 0.2628 & -0.0584 & 0.0795 \\
\hline
\end{tabular}

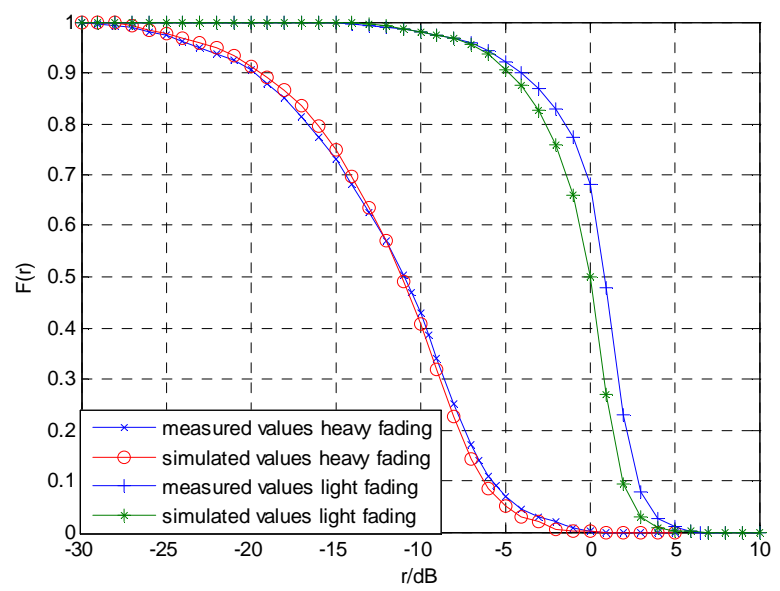

Fig.5. A comparison of measured and calculated values of CCDF

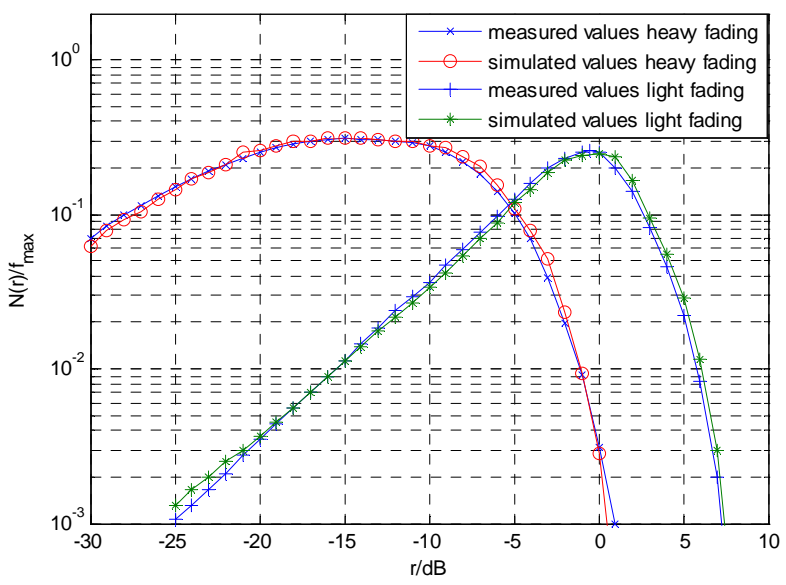

Fig.6. A comparison of measured and calculated values of LCR

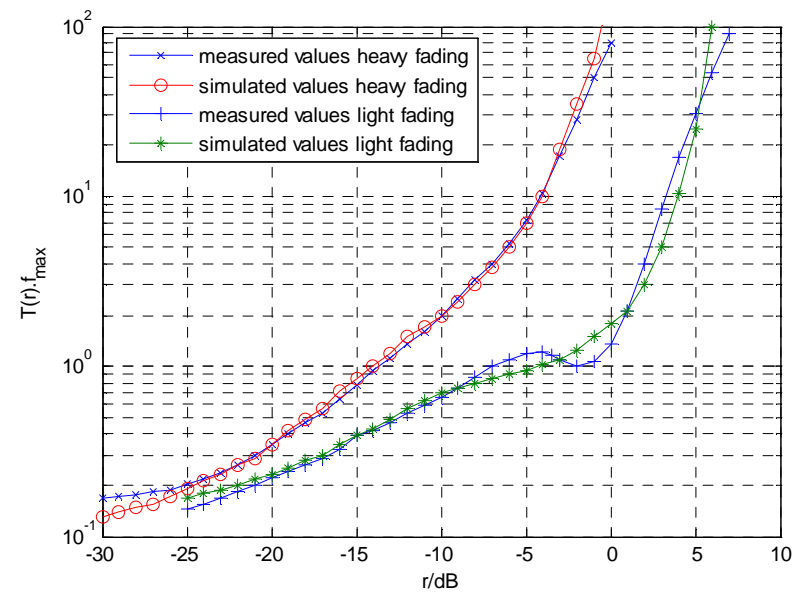

Fig.7. A comparison of measured and calculated values of AFD

Fig.5 Fig.7 presents numerical values for the complementary cumulative distribution function (CCDF), level crossing rate (LCR) and average fade duration (AFD) which is defined by Loo model given above. 
With the summary of the model above, GEO satellite mobile communication system channel model will be established as is indicated in Fig.8. Fading model switches among the three states in semi-Markov process.

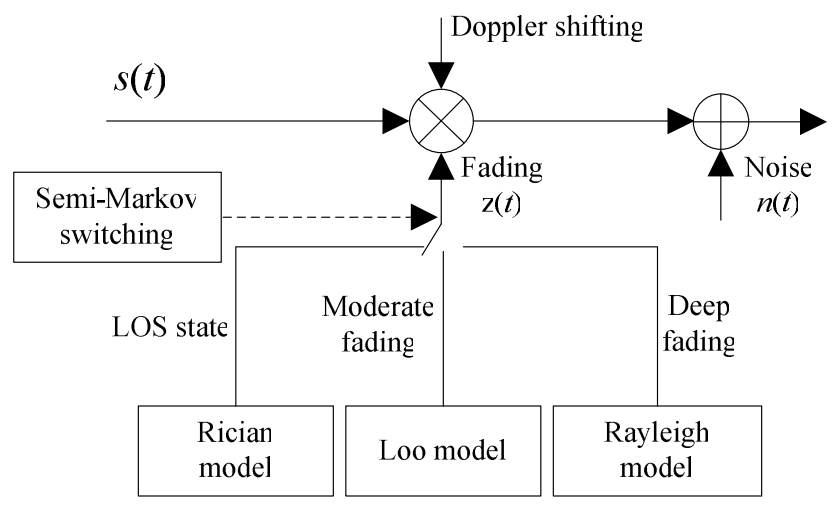

Fig.8. Channel simulation model

Tab.2. gives the measure results of parameters in satellite channel. Fig.9. shows the comparison of theoretical and simulated values of three states. At deep shadowing state, channel degenerate into Rayleigh fading; at light shadowing state, it is Rician fading with a certain fading factor; at moderate, channel state is between Rician and Rayleigh fading and similar to this fading at some time.

Tab.2. The measure results of parameters in satellite channel

\begin{tabular}{|c|c|c|c|}
\hline Fading degree & $0.5 \sigma_{\alpha}^{2}$ & $\mu_{z}$ & $\sigma_{z}$ \\
\hline light & 0.1580 & 0.115 & 0.115 \\
\hline moderate & 0.1260 & -0.115 & 0.161 \\
\hline deep & 0.0631 & -3.910 & 0.806 \\
\hline
\end{tabular}

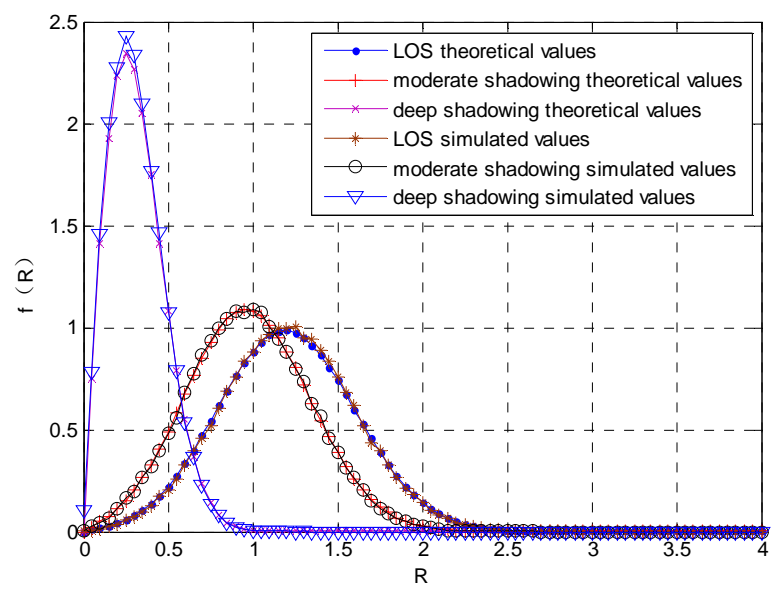

Fig.9. The comparison of theoretical and simulated values of three states

Fig.10. gives the signal power fading at different channel state, fading degree differs from each other, signal power at LOS state is $10 \sim 20 \mathrm{~dB}$ higher than at deep fading state.

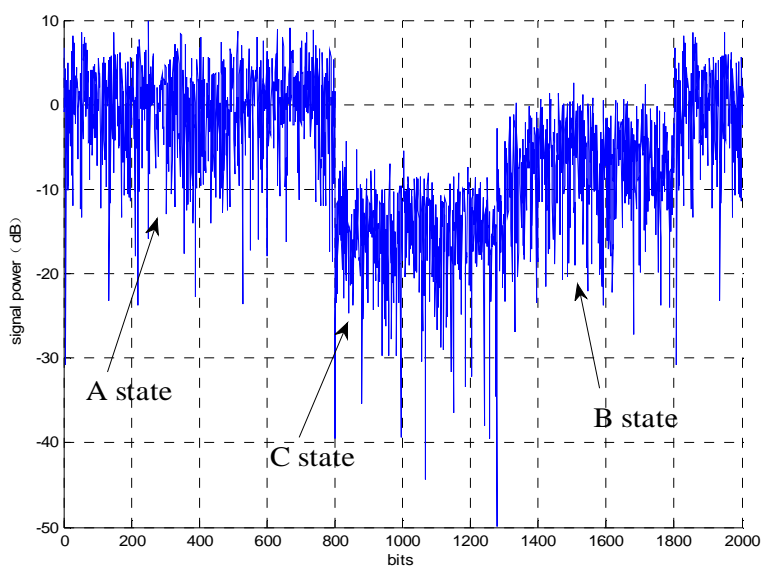

Fig.10. Fading signal power of three states

\section{CONCLUSION}

In this paper, a channel model based on three-state semi-Markov process is discussed. For LOS condition, the channel is described as Rician distribution and for deep shadowing condition is Rayleigh distribution. When in rural and suburb area, the channel state is under moderate shadowing condition and described as C.Loo model. The three states switches by semi-Markov process, each state remains about $5 \mathrm{~m}$, due to this result it allows us to describe the distribution of time duration. On the other hand, this channel model shows better properties than other systems. With the analysis of received signal PDF at the three states and the fading power of this model, it is possible to conclude that this model may be used in GEO satellite.

\section{REFERENCES}

[1] Yongjun Xie, Yuguang Fang. A general statistical channel model for mobile satellite systems[J]. IEEE Transactions on Vehicular Technology, 2000, 49(3):744-752.

[2] CHUN Loo. A Statistical Model for a Land Mobile Satellite Link[J]. IEEE Transactions on Vehicular Technology, 1985, 34(8): 122-127.

[3] Erich Lutz, Daniel Cygan, Michael Dippold, et al.. The Land Mobile Satellite Communication Channel-Recording, Statistics, and Channel Model[J]. IEEE Transactions on Vehicular Technology, 1991, 40(2): 375-386.

[4] Giovanni E. Corazza, Francesco Vatalaro. A statistical model for land mobile satellite channels and its application to nongeostationary orbit systems[J]. IEEE Transactions on Vehicular Technology, 1994, 43(3): 738-742.

[5] Fernando P F, Maryan V C, Cabado C E, et al. Statistical modeling of the LMS channel[J]. IEEE Transactions on Vehicular Technology, 2001, 50(6): 1549-1567.

[6] José Luis, Cuevas-Ruiz, José A. Delgado-Penín. Channel model based on semi-Markovian process. An approach for HAPS systems[C]. Proceedings of the $14^{\text {th }}$ International Conference on Electronics, Communication and Computers, 2004, 52-56.

[7] Recomendation ITU-R P.681-6.

[8] Branka Vucetic, Jun Du. Channel modeling and simulation in satellite mobile communication systems[J]. IEEE Journal on Selected Areas in Communications, 1992, 10(8): 1209-1218.

[9] Matthias Patzold. Mobile fading channels[M]. Publishing House of Electronics Industry, Beijing, 2009. 